\title{
Resistance Mechanism in a Terbinafine-Resistant Strain of Microsporum canis
}

\section{Rui Kano'1, Yun-Hsia Hsiao², Tsuyoshi Yamada³, Han Hock Siew', Charles Chen², Atsuhiko Hasegawa ${ }^{3}$ and Hiroshi Kamata ${ }^{1}$}

\author{
1'Department of Veterinary Pathobiology, Nihon University College of Bioresouce Sciences, 1866 \\ Kameino, Fujisawa, Kanagawa 252-0880, Japan \\ ${ }^{2}$ Asian Veterinary Specialist Referral Center, 1F, No. 109, Sec. 4, XingYi Rd. Taipei, 106, Taiwan \\ ${ }^{3}$ Teikyo University Institute of Medical Mycology, 359 Otsuka, Hachioji, Tokyo 192-0395, Japan \\ ${ }^{4} 42$ JLN Ponderosa 1/9, TMN Ponderosa, 81100 Johor Bahru, Johor, Malaysia
}

\section{Introduction}

Feline dermatophytosis is generally treated with azoles and terbinafine (TRF), compounds that demonstrate efficacy against both human and animal dermatophytoses. TRF is a member of the allylamine class of antifungal agents, which are known to target the squalene epoxidase (SQLE) enzyme in dermatophytes.

We first isolated a TRF-resistant Microsporum canis strain from a feline dermatophytosis in China. This strain (designated 47C) exhibited a TRF minimum inhibitory concentration (MIC) of $>32 \mu \mathrm{g} / \mathrm{mL}$ but remained susceptible to itraconazole (ITZ) (MIC $0.023 \mu \mathrm{g} / \mathrm{mL}$ ) (Table 1). To clarify the resistance mechanism in the $47 \mathrm{C}$ strain, the expression of the pleiotropic drug resistance (PDR1), multidrug resistance (MDR1), MDR2, and MDR4 genes were investigated by real-time quantitative PCR (RT-qPCR) analysis and sequenced SQLE encoding gene.

\section{Material and Methods}

Strains examined

Strains are summarized in Table 1. M. canis strains $12 \mathrm{C}, 13 \mathrm{C}$, and $14 \mathrm{C}$, which are known to be susceptible to both TRF and ITZ, were used as comparators (Table 1).

\section{$R T$ - $q P C R$ analysis of the $A B C$ transporter family genes}

Strains $12 \mathrm{C}, 13 \mathrm{C}, 14 \mathrm{C}$, and $47 \mathrm{C}$ were cultured for 4 days at $28{ }^{\circ} \mathrm{C}$ in Saboraud's dextrose broth (SDB; $1 \%$ peptone and $2 \%$ dextrose). TRF exposure was carried out by the previously reported method of Martins et al., who used a concentration of $0.14 \mu \mathrm{g} / \mathrm{mL}$ TRF to evaluate the expression of genes in 4 species of Trichophyton [1]. All strains (12C, $13 \mathrm{C}, 14 \mathrm{C}$, and $47 \mathrm{C}$ ) were cultured for 4 days at $28^{\circ} \mathrm{C}$ in SDB with and without $0.14 \mu \mathrm{g} / \mathrm{mL}$ TRF. Extraction for total RNAs from fungal cells and the reverse transcription procedures were reported [2].

To analyze the expression levels of the PDR1, MDR1, MDR2, and MDR4 genes, which encode members of the ABC transporter family, we used real-time quantitative PCR (RT-qPCR) were reported [2].

Sequence for SQLE encoding gene of TRF-resistent strain (47C) To sequence the SQLE cDNA from isolates, primers were prepared based on the conserved sequence of the Arthroderma otae CBS 113480 squalene epoxidase, mRNA (GenBank accession number, XM_002849385).

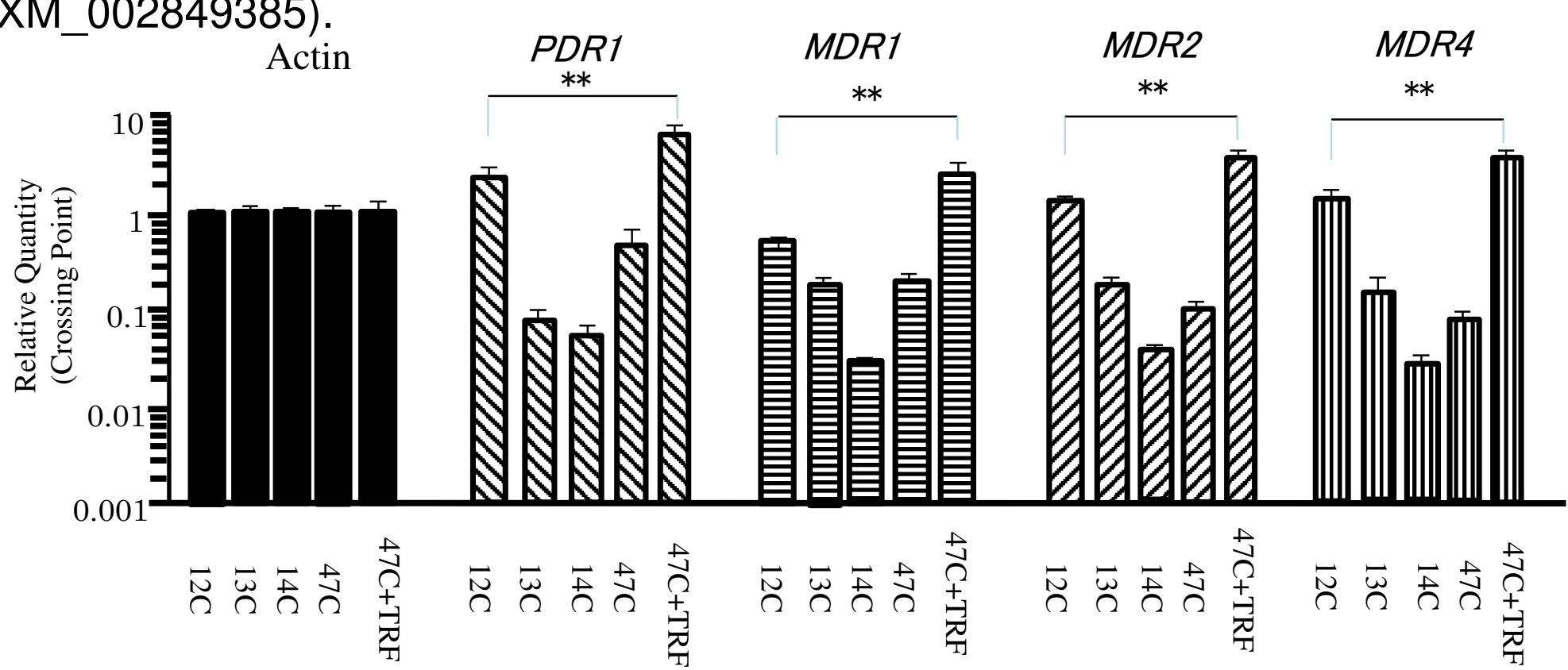

Fig. 1. Transcript levels of the actin-encoding gene and of PDR1, MDR1, MDR2, and MDR4 in TRF-resistant (47C) and -susceptible (12C, 13C, and 14C) strains of $M$. canis. All strains were cultured in SDB and (for $47 C$ only) in SDB containing $0.14 \mathrm{mg} / \mathrm{mL}$ TRF $(47 \mathrm{C}+\mathrm{TRF})$ for 4 days at $28^{\circ} \mathrm{C}$. Relative Quantity ( $\mathrm{y}$ axis) indicates that the expression levels of all genes were normalized to that of the actin-encoding gene in the respective strain. Asterisks indicate statistically significant differences by a two-tailed, non-paired $t$-test; ${ }^{* *} P<0.01$.

\section{Results}

Expression levels of PDR1, MDR1, MDR2, and MDR4

No significant differences were found in basal levels of actin expression among any of the strains cultured in SDB in the absence of TRF or compared to strain $47 \mathrm{C}$ cultured in SDB the presence of 0.14 $\mu \mathrm{g} / \mathrm{mL}$ of TRF (Fig. 1).

The transcript levels of PDR1, MDR1, MDR2, and MDR4 in the TRFresistant strain $(47 C)$ cultured in SDB were not significantly higher than those of the respective genes in the TRF-susceptible strains (12C, 13C, and 14C) (Fig. 1). However, the transcript levels of $P D R 1, M D R 1$, MDR2, and MDR4 in the TRF-resistant strain cultured in SDB containing $0.14 \mu \mathrm{g} / \mathrm{mL}$ of TRF were 2 to 4 times higher than those of the respective genes in the three TRF-susceptible strains (Fig.1).

\section{SQLE sequence of the TRF-resistant isolate}

The SQLE gene from the 47C strain encoded a protein with I100M and F395L substitutions (Fig. 2). The sequence determined in this study has been deposited in GenBank (Microsporum canis 47C SQEL mRNA for squalene epoxidase, complete cds; GenBank accession no. LC348388).

\section{Discussion}

To the best of our knowledge, this work represents the first report that TRF-resistant strains of $M$. canis exhibited the overexpression of $A B C$ transporter proteins and encodes missense mutations in the SQLE. We speculate that the overexpression of genes in the TRF-resistant strain is not sufficient to prevent the antifungal effects of ITZ. To investigate the relationship between these amino acid substitutions and TRF tolerance, point mutations leading to several amino acid substitutions found in the SQLE of the resistance isolate will be introduced into the endogenous SQLE gene of the $M$. canis using genetic manipulation tools.

Table $1 \mathrm{MICs}(\mathrm{mg} / \mathrm{mL})$ of anti-fungal drugs in the tested strains

\begin{tabular}{lccl}
\hline Strain & TRF $^{a}$ & ITZ $^{\mathrm{b}}$ & Origin \\
47C & $>32$ & 0.023 & feline dermatophytosis in China \\
$12 \mathrm{C}$ & 0.125 & 0.064 & feline dermatophytosis in Malaysia \\
$13 \mathrm{C}$ & 0.125 & 0.023 & feline dermatophytosis in Malaysia \\
$14 \mathrm{C}$ & 0.5 & 0.25 & Pet shop's clipper in Malaysia \\
\hline
\end{tabular}

aMICs were assayed by CLSI protocol M27-A3.

${ }^{\mathrm{b}} \mathrm{MICs}$ were assayed by E-test.

M. canis SQLE SVILLEKSWKEPDRIVGELLQPGGVRALEELGLRDCLEGIDAVRTYGYDVIYFGTGVKIP 12 $47 C$ SQLE

Canis SQLE SNTKLVLKOLSKFHWORKSLTSVINILAQALYSIFAAGDPNLKVLORGCFRIIDCLOGYT 420 47C SQLE

Fig. 2. Alignment of predicted $M$. canis SQLE amino acid sequence (Arthroderma otae CBS 113480 squalene epoxidase, mRNA; Gen Bank Accsession number:XM_002849385) with their counterpart from TRFresistent strain (47C). Amino acids identical to those of $47 \mathrm{C}$ SQLE are shown by asterisk.

\footnotetext{
References

Martins MP Franceschini AC, Jacob TR, Rossi A Martinez-Rossi NM Compensatory expression of multidrug-resistance genes encoding $A B C$ transporters in dermatophytes. J Med Microbiol. 2016;65:605-10.

2. Kano R, Hsiao YH, Han HS, Chen C, Hasegawa A, Kamata H. Resistance Mechanism in a Terbinafine-Resistant Strain of Microsporum canis. Mycopathologia. 2018 Jan 16. doi: 10.1007/s11046-018-0242-0.
} 